\title{
O Controle de Projetos Através dos Conceitos de Desempenho Real (Earned Value)
}

\author{
Oscar Fernando Osorio Balarine \\ Doutor em Engenharia de Produção \\ PUCRS - Av. Ipiranga, 6681-Prédio 40-Sala 502 \\ 90619-900 Porto Alegre(RS) \\ balarine@pucrs.br
}

\begin{abstract}
Resumo
Partindo dos conceitos de Administração de Projetos, este artigo objetiva mostrar a importância de um tipo especial de controle, auxiliar ao monitoramento de custos e tempos, visando alcançar os melhores resultados com projetos. É sugerida uma técnica de controle denominada Desempenho Real (Earned Value). Com isso, a totalidade do projeto pode ser constantemente controlado em seu progresso e desempenho, através da determinação de recursos necessários à conclusão dos trabalhos, mediante acompanhamento de seus custos e prazos.
\end{abstract}

Palavras-Chave: Administração de Projetos; Desempenho Real; Controle de Projetos.

\begin{abstract}
Starting from Project Management concepts, this paper aims to show a special kind of control method to aid costs and time monitoring to get better project results. It is suggested a control technique based on Earned Value. That is, the overall project can be constantly controlled in progress and performance through determination of resources required to complete the work, both in total and over time.
\end{abstract}

Keywords: Project Management; Earned Value; Project Control.

\section{Introdução}

A Administração de Projetos tem evoluído como tratamento diferenciado, dirigido à gestão de empreendimentos não-repetitivos. Popularizada nos anos 60 , com os programas espaciais norte-americanos, as técnicas de gestão de projetos vieram a consolidar-se, inicialmente aplicadas às indústrias petrolífera, química e da construção.

Hoje, sua aplicação estende-se para além dessas indústrias, em particular devido à quatro fortes razões, como indicado por Harrison (1993): (1) grande parte das atividades administrativas são projetos, exigindo tratamento diferenciado em sua gestão; (2) as condições de mercado tem reclamado maior profissionalismo dos agentes; (3) a aceleração da velocidade das mudanças demanda maior cuidado no tratamento dos objetivos de tempo, custos e desempenho; e (4) a integração multidisciplinar, e muitas vezes multiorganizacional, dentro das empresas, tem apontado para a adoção de técnicas de Administração de Projetos como referência para o alcance do sucesso esperado nos negócios.

De outro lado, algumas atividades empresariais são típicas estruturas voltadas para a Administração $P O R$ Projetos, como é o caso da atividade imobiliária, onde cada produto é, em si, um projeto.

Sem dúvida, atualmente as organizações vem se defrontando com problemas associados à gestão, que exigem o domínio de técnicas avançadas, auxiliares à realização de seus empreendimentos, com a habilidade e a competência exigidas. 
PRODUÇÃO

É neste sentido que este artigo pretende colaborar, descrevendo uma técnica simples e eficiente voltada para o controle de projetos, a partir de agora referenciada como DESEMPENHO REAL, título em Português que, entendese, melhor identifica a técnica internacionalmente conhecida como EARNED VALUE.

Para tanto, o artigo está dividido em seções, iniciando por esta introdução, seguida de breve descrição da Administração de Projetos. Posteriormente são analisados os papéis do planejamento e controle de projetos, com aplicabilidade da técnica de Desempenho Real, encerrando-se com comentários conclusivos.

\section{Administração de Projetos}

Entendidos como empreendimentos únicos e nãorepetitivos, os projetos comumente possuem duração determinada, são formalmente organizados e agregam recursos visando o cumprimento de objetivos préestabelecidos.

Burke (1997, p.9) define projeto como “...grupo de atividades que devem ser executadas em uma seqüência lógica, para alcançar objetivos determinados pelo cliente.", e Administração de Projetos, simplesmente como "...fazer o projeto acontecer".

Originalmente aceita como um ramo das Ciências da Administração, a Administração de Projetos evoluiu incorporando conhecimentos econômicos e de engenharia. Para isso, tratou do planejamento e controle de projetos, buscando otimizar tempos, custos e recursos. Iniciando com a concepção dos projetos em si (arquitetura), as técnicas incorporadas procuraram alcançar controle e otimização no tratamento das três restrições indicadas por Rosenau Jr. (1992), ou seja, (1) o desempenho de suas especificações, (2) no tempo programado, e (3) obedecendo aos custos orçados.

A consolidação da Administração de Projetos está associada aos programas espaciais norte-americanos na década de 60 (Kast \& Rosenzweig, 1980, p.216), com o refinamento de técnicas utilizadas para acompanhar e

integrar atividades, destacando-se os métodos PERT/CPM (Gray, 1981; Harrison, 1997), a Linha de Balanço (Scomazzon et al, 1985) e as Curvas de Agregação de Recursos (Heineck, 1989).

Hoje, a Administração de Projetos vem evoluindo com rapidez, inclusive recebendo a consideração de ciência distinta. Nos Estados Unidos, por exemplo, o Project Management Institute - PMI dedicou-se a definir um corpo de conhecimentos específicos para administrar projetos, através de um comitê de padronização (Stretton, 1990).

\subsection{Atributos Distintos ao Ambiente de Projetos}

Afirma-se que os projetos possuem atributos distintos da produção contínua, por serem: (1) únicos, ou seja, possuírem caminho próprio, tempo próprio, execução discreta, com um conjunto de objetivos claramente definidos e raramente repetitivos; e (2) finitos, ao possuírem início determinado e duração finita, além de conclusão e alcance de objetivos claramente definidos.

Destaca-se, também, que os projetos possuem atributos característicos (mas não exclusivos), como a multidisciplinaridade, a multiorganizacionalidade, uma complexidade particular e um dinamismo diferenciador em relação aos empreendimentos convencionais (Stretton, 1990, p.29).

Pondera-se, ainda, que estruturas organizacionais típicas exigem novas formas para tratamento dos projetos, dentre elas destacando-se o formato matricial que, apesar de multidepartamental, atribui responsabilidade centralizada em um gerente por projeto (Archibald, 1975).

Mas ainda que a Administração de Projetos trate de produto único e não-repetitivo e, portanto, relativamente diferenciador da produção contínua, suas técnicas observam a essência de administrar, através da aplicação dos conceitos clássicos, dentre os quais planejar e controlar. Assim, etapas dos projetos sempre recebem tratamento relativo (1) ao planejamento: pelo estabelecimento de metas e objetivos, com definições de 
suas tarefas e seqüências a realizar, com base nos recursos necessários e disponíveis; (2) ao controle: pelas medições de seu progresso e desempenho; e (3) às ações corretivas.

\subsection{Planejamento de Projetos}

Simplificadamente, pode-se definir planejar como organizar o futuro. Assim, o planejamento de um projeto consiste em processo que antecipe o que deve ser executado, através de identificação do trabalho a realizar em cada momento do tempo, levantando-se insumos e custos associados.

O processo consiste em coletar idéias e conhecimentos, organizando-os em pacotes de trabalho lógicos, dinâmica e continuamente, de forma a acompanhar a execução do projeto até sua conclusão, garantindo o alcance da satisfação de seus usuários finais.

Portanto, o processo de planejamento inicia com a descrição detalhada do produto (objetivos do projeto), seguida de programação do trabalho a realizar.

Posteriormente, levantam-se os custos de execução, materializados através de um orçamento.

Durante o estágio de planejamento, são realizadas a programação e o orçamento, como descrito a seguir:

a) Programação: utilizada para predizer os caminhos a adotar, associando-os aos tempos necessários de execução; a programação determina cada etapa e suas durações, até a conclusão do projeto, com a soma desses tempos definindo o prazo total de execução.

Inúmeras técnicas quantitativas são conhecidas visando programar projetos, desde as mais simples, como os Gráficos de Gantt (Diagramas de Barras), até aquelas técnicas mais sofisticadas envolvendo a composição de redes, como o PERT - Program Evaluation and Review Technique, surgido ao final dos anos 50, com o Projeto Polaris da Marinha dos Estados Unidos; ou o CPM - Critical Path Method, desenvolvido à mesma época pelas empresas Du Pont $\mathrm{e}$ Remington Rand Univac; ou, ainda, com a unificação de ambos, denominada PERT/CPM (Burke, 1997; Harrison,
1997; Hischfeld, 1985; Prado, 1988).

Este artigo não pretende detalhar tais técnicas, suficientemente conhecidas através de literatura direcionada e objeto de pacotes computacionais, como o software MS-Project, da Microsoft. Espera-se, isto sim, que a partir das informações obtidas através da programação do projeto, seja possível aplicar a técnica de controle denominada Desempenho Real, como na Seção 3, adiante, será enfocado.

b) Orçamento: complementar à programação, são estimados os custos exigidos, de forma detalhada e precisa, através da determinação dos recursos totais a utilizar em cada fase do projeto. Estas estimações irão permitir a distribuição dos ingressos e desembolsos associados às diversas etapas, em cada momento do tempo, gerando o fluxo de caixa do projeto. Também associado ao orçamento e seu fluxo de caixa, estarão as avaliações econômico-financeiras, através de considerações sobre a viabilidade do projeto (Balarine, 1997).

Neste artigo, também não serão detalhadas as técnicas orçamentárias, satisfatoriamente explicitadas na literatura (Caruso, 1980; Goldman, 1986; Sanvicente \& Santos, 1985).

Entende-se atinente, no entanto, uma breve explanação sobre o processo de organização do projeto, como será visto a seguir.

\subsection{Organização do Projeto}

Visando organizar a execução de um produto, Duncan (1993) identifica três processos administrativos básicos: (1) o planejamento, antecipando programas de trabalho destinados a atingir objetivos pré-determinados; (2) a execução, que traduz-se na realização dos planos; e (3) o controle, representado pelo monitoramento contínuo do progresso executivo, acompanhado das ações corretivas necessárias.

Dentre os três processos, aquele autor destaca as relações típicas do planejamento do empreendimento, como visto na Figura 1. 


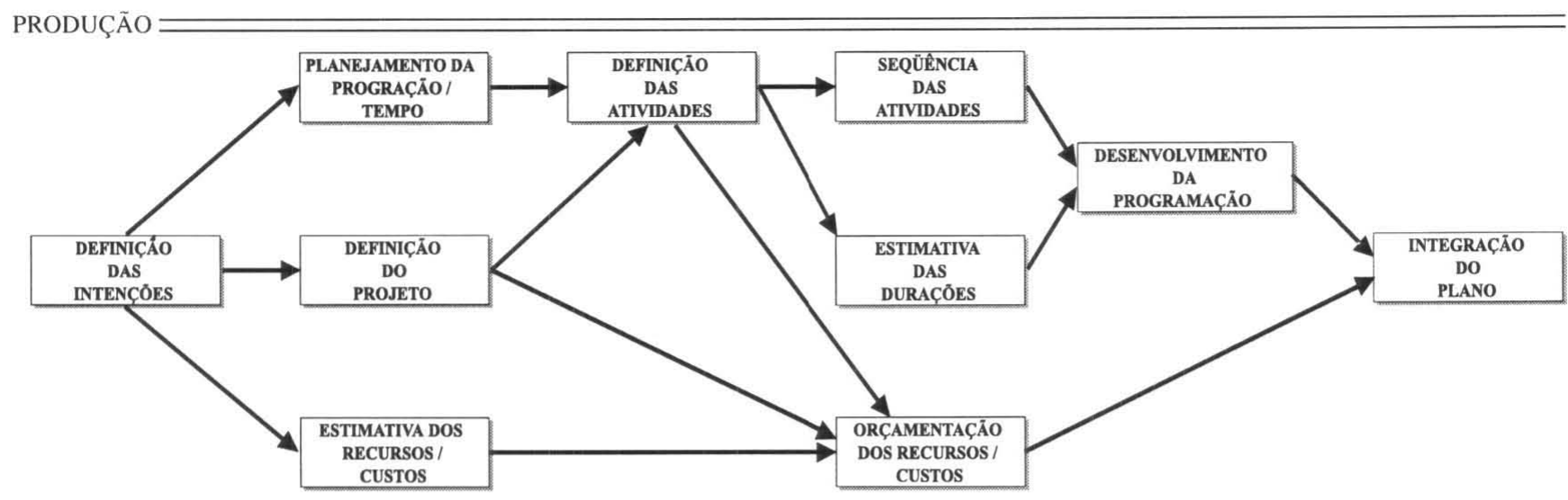

Fonte: Duncan, 1993, p.7

Figura 1. Planejamento do Empreendimento - Processos Dependentes

Tais processos de planejamento dependentes, incluem:

a) definição das intenções: justificativa do projeto, através de documento escrito descrevendo as intenções e objetivos a alcançar;

b) definição do projeto: decomposição das metas a atingir, de forma detalhada e que proporcione melhor controle;

c) definição das atividades: identificação das atividades necessárias ao desempenho desejado, para alcançar os objetivos finais;

d) sequiência das atividades: identificação das dependências entre as diversas atividades;

e) estimativa das durações: estimação das durações prováveis das atividades e tarefas;

f) desenvolvimento da programação: determinação das datas para as atividades;

g) estimativa de custos: desenvolvimento inicial dos custos totais do projeto;

h) orçamentação de custos: desenvolvimento detalhado das estimações de custos para cada atividade; e

i) integração do plano: idealização documentada de um plano coerente para o projeto como um todo.

Cada um dos processos anteriormente descritos será associado ao ciclo de vida do projeto, com suas interações claramente identificadas e monitoradas, através de gerenciamento ativo.

\subsection{Controle de Projetos}

Distribuídas seqüencialmente as etapas necessárias à execução do projeto e determinados seus custos associados, é o momento de utilizar cada recurso exigido (recursos humanos, financeiros, materiais, equipamentos, etc.), para execução e obtenção do produto final projetado.

No entanto, ainda que detalhadamente planejado, à medida que o projeto evolua, irão surgir desvios dos planos, originados em erros, omissões ou modificações não previstas.

Assim, durante todo o ciclo de vida do projeto será necessário acompanhamento criterioso de sua execução, através da análise de informações que permitam comparar o progresso atual com a programação, custos e recursos estabelecidos no plano inicial. É o momento, pois, do processo de controle tornar-se dominante, na evolução da administração do projeto.

É importante salientar que, como sugerido por Harrison (1993, p.97), no trabalho com projetos, planejamento e controle não são funções discretas e separadas. Elas interagem uma com a outra e são interdependentes, num ciclo contínuo em que o planejamento produz informações necessárias ao controle, ao mesmo tempo em que o controle realimenta o planejamento. 


\section{Desempenho Real (Earned Value)}

Revistos os conceitos elementares de planejamento e controle aplicados à Administração de Projetos, é o momento de descrever a técnica bastante simples e eficiente direcionada ao controle de projetos, conhecida internacionalmente por Earned Value. Entendese que, em Português, as palavras com melhor significado para tradução (não-literal) daquela técnica, sejam Desempenho Real, como aqui vem sendo referenciado. Para tanto, inicia-se esta Seção com a descrição das Curvas de Agregação de Recursos, que auxiliam o entendimento daquela técnica, para depois caracterizar o controle por Desempenho Real. Encerra-se a Seção com exemplo aplicativo simulado.

\subsection{Curvas de Agregação de Recursos}

As Curvas de Agregação de Recursos representam técnica de planejamento, programação e controle de projetos, consistindo simplesmente na agregação (soma ou totalização) dos recursos utilizados em um projeto, período a período. Tais recursos podem ser "...homens-hora, volume ou quantidade de materiais, número de máquinas, ou o denominador comum de todos estes, ou seja, o valor em ..." Reais, CUBs ${ }^{1}$, Dólares Norte-Americanos, etc., investidos no projeto (Heineck, 1989, p.1).

A curva é desenhada sob a forma de gráfico, num sistema cartesiano, com o tempo distribuído no eixo $\mathrm{x}$ (podem ser dias, semanas, meses ou anos); enquanto que os recursos são registrados no eixo y.

Uma derivação particular da Curva de Agregação de Recursos, que interessa particularmente à introdução da técnica de Desempenho Real, é aquela em que o tempo e os recursos são transformados em percentuais acumulados, gerando as chamadas Curvas "S" (Formoso et al, 1986), representativas da distribuição dos recursos utilizados associados ao tempo decorrido, conforme exemplificado na Figura 2.

\section{Curva "S" Típica}

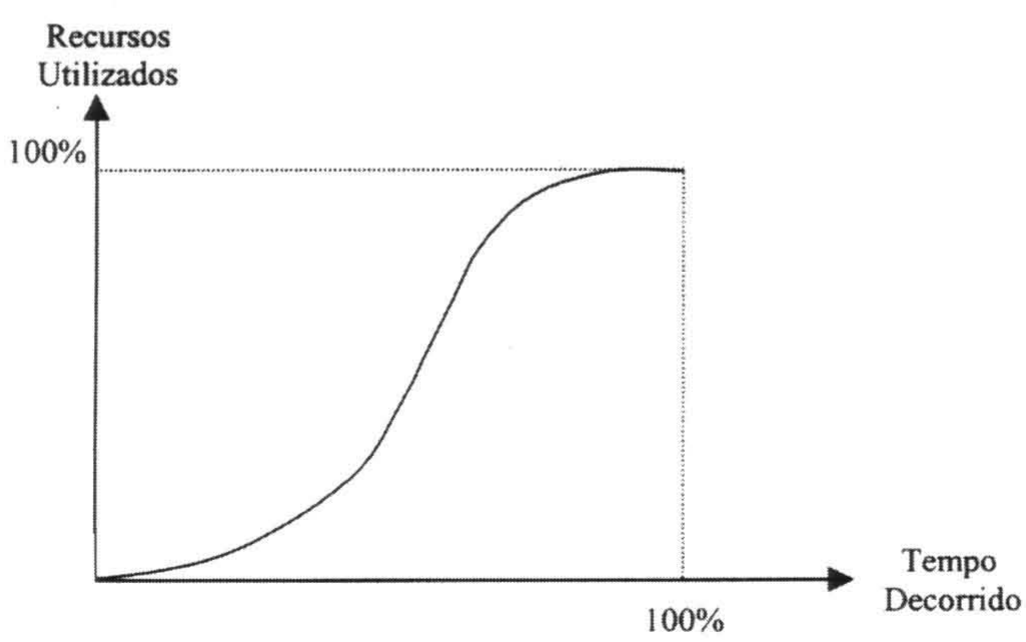

Figura 2

\footnotetext{
' CUB - Custo Unitário Básico: unidade de medida do custo de um metro quadrado de construção, levantado pelos sindicatos regionais da Indústria da Construção Civil, conforme Lei Federal No. 4.591 e NBR 12.721/93, da ABNT.
} 
PRODUÇÃO

Como visto, a Curva " $\mathrm{S}$ " inicia apresentando concavidade para cima, atinge seu ponto de inflexão (tempo relativo em que é realizado o maior volume de consumo do recurso considerado); a partir daí, e até o momento final da atividade retratada, registra concavidade para baixo.

Pindyck \& Rubinfeld (1991, p.422) apresentam função geradora das Curvas "S", que podem ser obtidas a partir da seguinte equação:

$Y_{t}=e^{k 1-(k 2 / t)}$

onde: e = Logarítmo Neperiano;

k1 e k2 = Parâmetros.

\subsection{Desempenho Real (Earned Value)}

Tradicionalmente, o controle dos "recursos versus tempos" consumidos em projetos, tem sido acompanhado através da Análise de Variância. A utilização das Curvas de Agregação de Recursos podem ser plotadas, então, comparando-se o custo originalmente orçado com o custo atual efetivamente realizado, à medida em que o projeto evolua, como ilustrado à Figura 3.

Com base neste conceito, são acompanhadas as diferenças entre o progresso atual do projeto e o plano original, gerando variâncias que podem ser positivas ou negativas.

Simplificadamente, poderá ilustrar-se a situação descrita, tomando como exemplo determinado projeto destinado à construção de edificação, com as seguintes características:

a) Custo Orçado (CO) total: $\$ 1.000$ (mil unidades monetárias);

b) Prazo para execução da edificação: 50 semanas.

Considere-se, agora, que após decorrido determinado tempo em que o projeto, como originalmente orçado (CO), deveria ter consumido $\$ 410$, apresente, na verdade, um Custo Atual (CA) de \$425. Conforme o conceito de variância, o quadro descrito indicaria, apenas, que, na data da medição, o Custo Atual (CA) estaria ultrapassando o Custo Orçado (CO) em \$15. Nenhuma outra indicação seria obtida, além da constatação de que os desembolsos ultrapassaram o orçamento. Portanto, a Análise de Variância é bastante limitada, destacando-se as seguintes restrições:

a) trata-se de técnica puramente histórica, não

\section{Custo Orçado (CO) x Custo Atual (CA)}

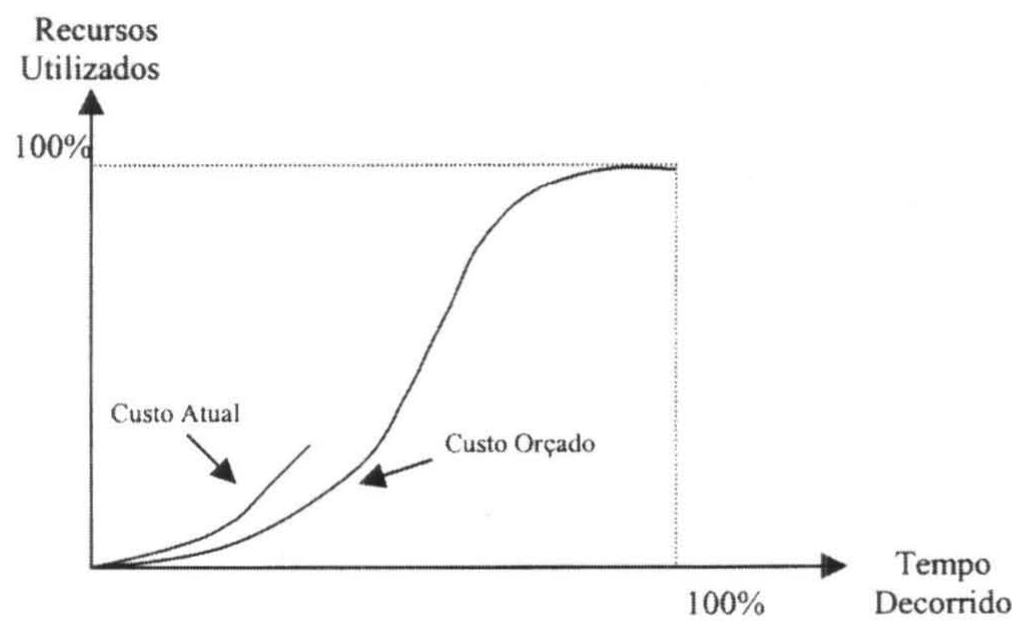

Figura 3 
vislumbrando ocorrências futuras;

b) não utiliza a potencialidade de todos os dados disponíveis;

c) não indica tendências; e

d) deixa de integrar informações de custos, com informações sobre o tempo decorrido.

Visando suprir tais lacunas, é sugerida a utilização da técnica de Desempenho Real (Earned Value), metodologia que considera custos e prazos de forma integrada, através da associação de um terceiro elemento à análise, correspondendo ao Custo Orçado (CO) como função dos custos do trabalho atualmente concluído (Town, 1998). Portanto, o Desempenho Real é identificado pelo que foi fisicamente obtido, proporcionalmente ao custo até então incorrido (Fleming \& Koppelman, 1996, p.130).

Retornando ao exemplo do projeto de edificação anteriormente suposto, e, agora, incluindo nova informação correspondente ao trabalho atual executado, quantificado conforme custos originais orçados, seriam obtidos os seguintes dados:
a) Custo Orçado (CO) total: $\$ 1.000$;
b) prazo total: 50 semanas;
c) posição planejada para o Custo Orçado, à data da medição: \$410;

d) Desempenho Real (Earned Value): \$375.

Então, de posse desses dados, podem ser geradas as seguintes informações auxiliares à tomada de decisão:

$1^{\circ}$.) Em relação aos CUSTOS:

a) Variância nos custos: $375-425=-50$

Constatação: o projeto está $\$ 50$ acima do orçado.

b) Desempenho de custos: $375 / 425=0,88$

Constatação: foram executados apenas $88 \%$ do planejado. c) Custo final projetado: $1.000 / 0,88=1.136$

Constatação: o projeto terá um sobrecusto de $\$ 136^{2}$.

$2^{\circ}$.) Em relação aos PRAZOS:

a) Variância no tempo (como função dos custos): 375

$-410=-35$

Constatação: o projeto está atrasado em \$ 35 .

b) Desempenho: $375 / 410=0,915$

Constatação: foram executados $91,5 \%$ do planejado.

c) Prazo final projetado: 50/0,915 = 54,6

Constatação: o projeto atrasará 4,6 semanas ${ }^{3}$.

As curvas correspondentes aos três elementos utilizados na análise, podem ser observadas na Figura 4 .

\section{Conclusões}

Este artigo visou divulgar técnica conhecida internacionalmente como Earned Value, aqui chamada de Desempenho Real. Iniciando por caracterizar a Administração de Projetos, o estudo incursionou pelo planejamento e controle, posteriormente incorporando os conceitos das Curvas de Agregação de Recursos e do Desempenho Real, ilustrados por gráficos e exemplo simulado.

Como visto, o processo é simples e eficiente, mostrando utilidade especial para auxiliar a análise e monitoramento de projetos em execução.

Surpreende, no entanto, que a técnica de Desempenho Real, conhecida desde os anos 60, não venha sendo adotada por empresas nacionais que trabalham com projetos.

Considerando que o método potencializa obter informações preciosas sobre custos e prazos, de forma simples e eficaz, espera-se que este artigo sirva como introdução ao tema, provocando curiosidade naqueles que dedicam-se ao planejamento e controle de projetos. Então, a bibliografia indicada poderá servir como referência para

\footnotetext{
${ }^{2,3}$ Projeções com base no desempenho atual, desconsideradas interferências no rítmo de execução do projeto.
} 
PRODUÇÃO

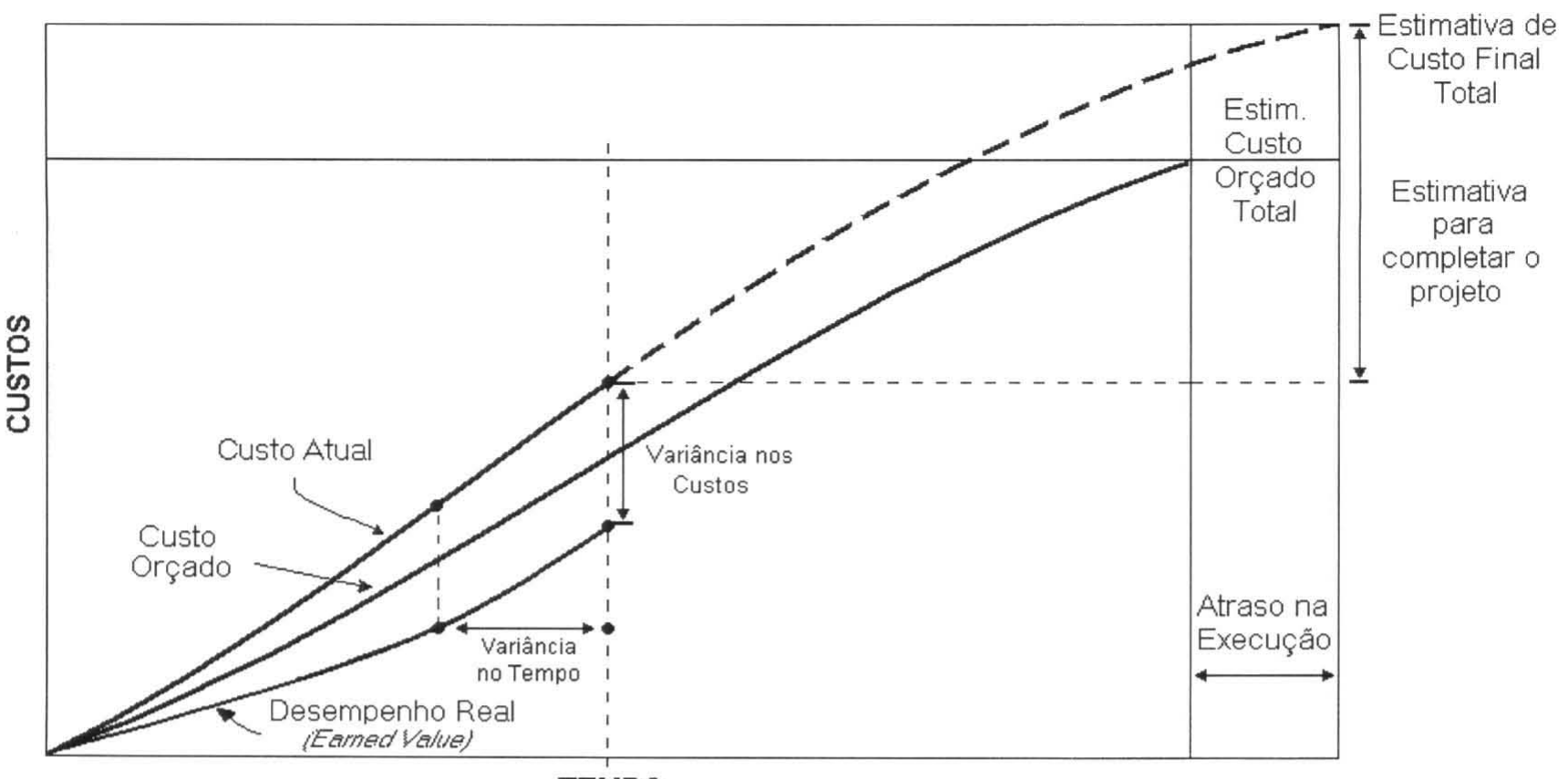

Figura 4

maior aprofundamento sobre essa útil ferramenta, auxiliar à tomada de decisão.

Nutre-se a esperança de que, adotado o conceito de Desempenho Real, através de pequeno aumento no tempo dedicado ao processo de planejamento e controle, sejam proporcionados expressivos acréscimos à probabilidade de sucesso dos projetos.

\section{Referências Bibliográficas}

ARCHIBALD, R. Managing High-Technology Programs and Projects. New York, Willey-Interscience, 1976.

BALARINE, O. Contribuições Metodológicas ao Estudo de Viabilidade Econômico-Financeira das Incorporações Imobiliárias. In: FORMOSO, C. (Ed.). Métodos e Ferramentas para a Gestão da Qualidade e Produtividade na Construção Civil. Porto Alegre, UFRGS-NORIE/PQPCCRS, 1997. pp.11-28.
BURKE, R. Project Management: Planning and Control. $2^{\text {nd }}$. Ed. Chichester, John Wiley \& Sons, 1997.

CARUSO, C. Orçamento na Teoria e na Prática. São Paulo, Centro Técnico de Especialização, 1980.

DUNCAN, W. The Process of Project Management. Project Management Journal. V. XXIV, N.3, September 1993. pp.5-10.

FLEMING, Q. \& KOPPELMAN, J. Earned Value Project Management. Upper Darby, Project Management Institute, 1996.

FORMOSO, C.; HIROTA, E.; SAFFARO, F. \& SILVA, M. Estimativa de Custos de Obras de Edificação. Caderno de Engenharia No. 9. Porto Alegre, UFRGS/CPGEC, 1986.

GOLDMAN, P. Introdução ao Planejamento e Controle de Custos na Construção Civil. São Paulo, Pini, 1986. 
GRAY, C. Essentials of Project Management. Princeton, Petrocelli, 1981.

HARRISON, A. A Survival Guide to Critical Path Analysis and the Activity on Node Method. Oxford, Butterworth-Heinemann, 1997.

HARRISON, F. Advanced Project Management: a Structured Approach. $3^{\text {rd }}$. Ed. Hampshire, Gower, 1993.

HEINECK, L. Curvas de Agregação de Recursos no Planejamento e Controle da Edificação: Aplicações a Obras e a Programas de Construção. Caderno de Engenharia No. 31. Porto Alegre, UFRGS/CPGEC, 1989.

HIRSCHFELD, H. Planejamento com PERT/CPM. $8^{\mathrm{a}}$. Ed. São Paulo, Atlas, 1985.

KAST, F. \& ROSENZWEIG, J. Organização e Administração: um Enfoque Sistêmico. $2^{\text {a }}$. Ed. 2v. São Paulo, Pioneira, 1980.

PRADO, D. Administração de Projetos com PERT/CPM. Belo Horizonte, Editora UFMG, 1988.

PINDYCK, R. \& RUBINFELD, D. Econometric Models and Economic Forecasts. $3^{\text {rd }}$. Ed. New York, McGrawHill, 1991.

ROSENAU JR., M. Successful Project Management. $2^{\text {nd }}$. Ed. New York, Van Nostrand Reinhold, 1992.

SANVICENTE, A. \& SANTOS, C. Orçamento na

Administração de Empresas. 2a. Ed. São Paulo, Atlas, 1985.

SCOMAZZON, B.; SOIBELMAN, L. \& SILVA, N.

Planejamento, Programação e Controle de Obras Repetitivas: Técnica da Linha de Balanço - Estudo de Caso. Caderno de Engenharia No. 13. Porto Alegre, UFRGS/CPGEC, 1985.
STRETTON, A. A Body of Knowledge for Project Management and Beyond? CIB 90. Proceedings... 1421 March 1990. V.4. pp.27-42.

TOWN, C. Project Control and Earned Value Management. Management Accounting. V.76, N.8, September 1998. pp.22-4. 